\title{
Adaptive neuro-fuzzy inference system for prediction of water level in reservoir
}

\author{
Fi-John Chang *, Ya-Ting Chang \\ Department of Bioenvironmental Systems Engineering and Hydrotech Research Institute, \\ National Taiwan University, Roosevelt Road, Taipei 10770, Taiwan, ROC
}

Received 7 April 2004; received in revised form 23 March 2005; accepted 28 April 2005

Available online 15 July 2005

\begin{abstract}
Accurate prediction of the water level in a reservoir is crucial to optimizing the management of water resources. A neuro-fuzzy hybrid approach was used to construct a water level forecasting system during flood periods. In particular, we used the adaptive network-based fuzzy inference system (ANFIS) to build a prediction model for reservoir management. To illustrate the applicability and capability of the ANFIS, the Shihmen reservoir, Taiwan, was used as a case study. A large number (132) of typhoon and heavy rainfall events with 8640 hourly data sets collected in past 31 years were used. To investigate whether this neuro-fuzzy model can be cleverer (accurate) if human knowledge, i.e. current reservoir operation outflow, is provided, we developed two ANFIS models: one with human decision as input, another without. The results demonstrate that the ANFIS can be applied successfully and provide high accuracy and reliability for reservoir water level forecasting in the next three hours. Furthermore, the model with human decision as input variable has consistently superior performance with regard to all used indexes than the model without this input.

(C) 2005 Elsevier Ltd. All rights reserved.
\end{abstract}

Keywords: Adaptive neuro-fuzzy inference system (ANFIS); Artificial neural networks; Water level forecasting; Reservoir management; Control and prediction

\section{Introduction}

Reservoirs are the most important and effective water storage facilities in modifying uneven distribution of water both in space and time. They not only provide water, hydroelectric energy and irrigation, but also smooth out extreme inflows to mitigate floods or droughts. To make the best use of the available water, the optimal operation of reservoirs in a system is undoubtedly very important. Reservoir operation requires a series of decisions that determine the accumulation and release of water over time. In the face of natural

\footnotetext{
${ }^{*}$ Corresponding author. Fax: +886 223635854.

E-mail address: changfj@ntu.edu.tw (F.-J. Chang).
}

uncertainty, forecasts of future reservoir inflow can be helpful in making efficient operating decisions.

With the high mountains and steep slopes of Taiwan, heavy rainfall, especially in a typhoon event, could cause downstream flooding within a few hours. Nevertheless, the typhoon events, usually coupled with abundant rainfall, are the most valuable yearly water resource. Accurate prediction of inflow rates is crucial not only for optimizing the management of water resources but for sustaining the safety of a reservoir. For decades, a wide variety of approaches have been proposed for streamflow forecasting including statistical (black-box) and physical (conceptual) models (e.g. [9,11,32]). Owing to the strongly non-linear, high degree of uncertainty, and time-varying characteristics of the hydrosystem, none of them can be considered as a single superior model [34]. An accurate site-specific prediction remains a difficult task. 
Recently, artificial neural networks (ANNs) have been accepted as a potentially useful tool for modeling complex non-linear systems and widely used for prediction [16]. In the hydrological forecasting context, ANNs have also proven to be an efficient alternative to traditional methods for rainfall forecasting $[13,15,26]$, streamflow forecasting $[2-4,6,10,18,22,36,39]$, groundwater modeling [24,37], and reservoir operation $[17,19,30]$. The ASCE Task Committee report [40,41] did a comprehensive review of the application of ANNs to hydrology, as did Maier and Dandy [27], and also Govindaraju and Rao [14] in a specialized publication.

In this study, we present a novel neuro-fuzzy approach, namely adaptive neuro-fuzzy inference system (ANFIS), in forecasting 1-3 hours-ahead water level of a reservoir during flood periods. To verify its applicability, the Shihmen reservoir, Taiwan, was chosen as the study area. Because the reservoir water level is a control system, its variability cannot be solely determined by meteorological effects. Human knowledge and its operating decisions could significantly change the status of water level within a short period; consequently, a suitable prediction model should include upstream conditions and human decisions. To demonstrate that the neuro-fuzzy network has the ability to deal with human knowledge and enhance the model performance, we developed two ANFIS models for water level forecasting, one with a human decision of reservoir outflow as input variable, another without.

In the following, the methodology of constructing the ANFIS model for prediction of the reservoir water level is presented. The theorem, network structure, and parameters estimating algorithms are described first. Next, a presentation of the study watershed, available data, and model construction are given. In Section 4, the results of two ANFIS model are presented and discussed. Last, a conclusion of this study is drawn.

\section{The methodology of ANFIS}

Since Zadeh [38] proposed the fuzzy logic theorem to describe complicated systems, it has became very popular and been successfully used in various problems, especially on control processes such as chemical reactors, automatic trains and nuclear reactors. More recently, fuzzy logic has been highly recommended for modeling reservoir operation to solve the inherent imprecision and vagueness characteristics in reservoirs [23,31]. Nevertheless, the main problem with fuzzy logic is that there is no systematic procedure for the design of a fuzzy controller. On the other hand, a neural network has the ability to learn from the environment (input-output pairs), self-organize its structure, and adapt to it in an interactive manner. For this reason, we propose the use of the adaptive neuro-fuzzy inference system (ANFIS) methodology [21] to self-organize network structure and to adapt parameters of the fuzzy system for predicting the water level of a control reservoir system.

\subsection{Architecture and algorithm}

The ANFIS is a multilayer feedforward network which uses neural network learning algorithms and fuzzy reasoning to map an input space to an output space. With the ability to combine the verbal power of a fuzzy system with the numeric power of a neural system adaptive network, ANFIS has been shown to be powerful in modeling numerous processes, such as motor fault detection and diagnosis [1], power systems dynamic load [12,28], wind speed [33], forecasting system for the demand of teacher human resources [25], and real time reservoir operation $[5,7,29]$.

ANFIS possesses good capability of learning, constructing, expensing, and classifying. It has the advantage of allowing the extraction of fuzzy rules from numerical data or expert knowledge and adaptively constructs a rule base. Furthermore, it can tune the complicated conversion of human intelligence to fuzzy systems. The main drawback of the ANFIS predicting model is the time requested for training structure and determining parameters, which took much time.

For simplicity, we assume the fuzzy inference system under consideration has two inputs, $x$ and $y$, and one output, $z$. For a first-order Sugeno fuzzy model [35], a typical rule set with two fuzzy if-then rules can be expressed as

Rule 1: If $x$ is $A_{1}$ and $y$ is $B_{1}$ then $z_{1}=p_{1} * x+q_{1} * y+r_{1}$ Rule 2: If $x$ is $A_{2}$ and $y$ is $B_{2}$ then $z_{2}=p_{2} * x+q_{2} * y+r_{2}$

where $p_{i}, q_{i}$ and $r_{i}(i=1$ or 2$)$ are linear parameters in the then-part (consequent part) of the first-order Sugeno fuzzy model. The architecture of ANFIS consists of five layers (Fig. 1), and a brief introduction of the model is as follows.

Layer 1: input nodes. Each node of this layer generates membership grades to which they belong to each of the appropriate fuzzy sets using membership functions.

$$
\begin{aligned}
& O_{1, i}=\mu_{A_{i}}(x) \quad \text { for } i=1,2 \\
& O_{1, i}=\mu_{B_{i-2}}(y) \quad \text { for } i=3,4
\end{aligned}
$$

where $x, y$ are the crisp inputs to node $i$, and $A_{i}, B_{i}$ (small, large, etc.) are the linguistic labels characterized by appropriate membership functions $\mu_{A_{i}}, \mu_{B_{i}}$, respectively. Due to smoothness and concise notation, the Gaussian and bell-shaped membership functions are increasingly popular for specifying fuzzy sets. The bellshaped membership functions have one more parameter 


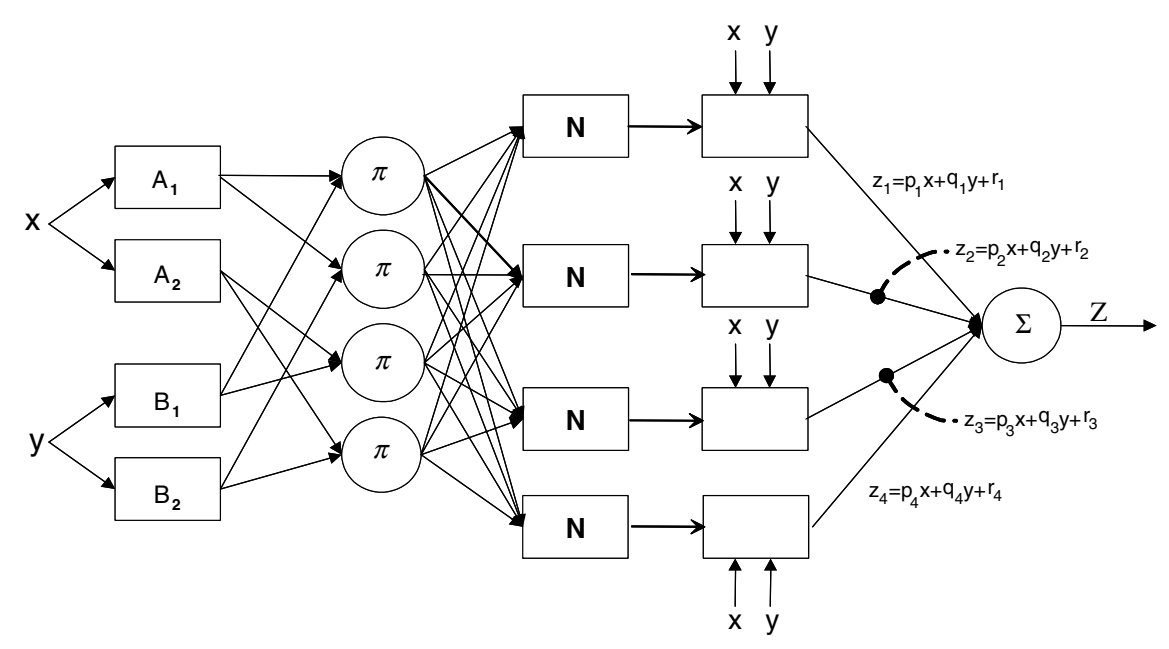

Fig. 1. ANFIS architecture for two-input Sugeno fuzzy model with four rules.

than the Gaussian membership functions, so a nonfuzzy set can be approached when the free parameter is tuned. The bell-shaped membership function is used in this study

$$
\mu_{A_{i}}=\frac{1}{1+\left|\frac{x-c_{i}}{a_{i}}\right|^{2 b_{i}}} \quad \mu_{B_{i-2}}=\frac{1}{1+\left|\frac{y-c_{i}}{a_{i}}\right|^{2 b_{i}}}
$$

where $\left\{a_{i}, b_{i}, c_{i}\right\}$ is the parameter set of the membership functions in the premise part of fuzzy if-then rules that changes the shapes of the membership function. Parameters in this layer are referred to as the premise parameters.

Layer 2: rule nodes. In the second layer, the AND operator is applied to obtain one output that represents the result of the antecedent for that rule, i.e., firing strength. Firing strength means the degrees to which the antecedent part of a fuzzy rule is satisfied and it shapes the output function for the rule. Hence the outputs $O_{2, k}$ of this layer are the products of the corresponding degrees from Layer 1

$$
\begin{aligned}
& O_{2, k}=w_{k}=\mu_{A_{i}}(x) \times \mu_{B_{j}}(y) \quad k=1, \ldots, 4 ; \\
& \quad i=1,2 ; j=1,2
\end{aligned}
$$

Layer 3: average nodes. In the third layer, the main objective is to calculate the ratio of each ith rule's firing strength to the sum of all rules' firing strength. Consequently, $\overline{w_{i}}$ is taken as the normalized firing strength

$O_{3, i}=\overline{w_{i}}=\frac{w_{i}}{\sum_{k=1}^{4} w_{k}} \quad i=1, \ldots, 4$

Layer 4: consequent nodes. The node function of the fourth layer computes the contribution of each ith rule's toward the total output and the function defined as

$O_{4, i}=\bar{w}_{i} f_{i}=\overline{w_{i}}\left(p_{i} x+q_{i} y+r_{i}\right), \quad i=1, \ldots, 4$ where $\overline{w_{i}}$ is the $i$ th node's output from the previous layer. As for $\left\{p_{i}, q_{i}, r_{i}\right\}$, they are the coefficients of this linear combination and are also the parameter set in the consequent part of the Sugeno fuzzy model.

Layer 5: output nodes. The single node computes the overall output by summing all the incoming signals. Accordingly, the defuzzification process transforms each rule's fuzzy results into a crisp output in this layer

$O_{5,1}=\sum_{i=1}^{4} \overline{w_{i}} f_{i}=\frac{\sum_{i=1}^{4} w_{i} f_{i}}{\sum_{i=1}^{4} w_{i}}$

This network is trained based on supervised learning. So our goal is to train adaptive networks to be able to approximate unknown functions given by training data and then find the precise value of the above parameters.

The distinguishing characteristic of the approach is that ANFIS applies a hybrid-learning algorithm, the gradient descent method and the least-squares method, to update parameters. The gradient descent method is employed to tune premise non-linear parameters $\left(\left\{a_{i}, b_{i}\right.\right.$, $\left.c_{i}\right\}$ ), while the least-squares method is used to identify consequent linear parameters $\left(\left\{p_{i}, q_{i}, r_{i}\right\}\right)$. As seen in Fig. 1, the circular nodes are fixed (i.e., not adaptive) nodes without parameter variables, and the square nodes have parameter variables (the parameters are changed during training). The task of the learning procedure has two steps: In the first step, the least square method to identify the consequent parameters, while the antecedent parameters (membership functions) are assumed to be fixed for the current cycle through the training set. Then, the error signals propagate backward. Gradient descent method is used to update the premise parameters, through minimizing the overall quadratic cost function, while the consequent parameters remain fixed. The detailed algorithm and mathematical background of the hybrid-learning algorithm can be found in [21]. 


\section{Study watershed and modeling}

To illustrate the practical application of the ANFIS forecasting model, the Shihmen reservoir, Taiwan, is used as a case study. The Shihmen reservoir is situated upstream of the Tahan Creek. The reservoir operates for multiple purposes such as irrigation, industrial and domestic use, flood control, hydropower generation, and recreation facilities. The Shihmen reservoir watershed occupies $763.4 \mathrm{~km}^{2}$ and reservoir effective storage is about $2.35 \times 10^{8} \mathrm{~m}^{3}$. The high water level of Shihmen reservoir is at $245 \mathrm{~m}$, while the dead water level is at $195 \mathrm{~m}$. The reservoir makes use of a saddle-chute spillway that has six control gates and can discharge floodwater at a maximum rate of $11,400 \mathrm{~m}^{3} / \mathrm{s}$. Besides, the discharge capacity of the two tunnel spillways is $2400 \mathrm{~m}^{3} / \mathrm{s}$. When a typhoon is expected, the water level in the dam can be reduced in advance to increase its floodwater storage capacity.

\subsection{The available data}

There are five water level gauge stations, which are equipped with automatic water level recorders and transmitted by wireless, above the Shihmen reservoir (Fig. 2 and Table 1). The hourly water level of the reservoir and the upstream five gauge stations are used. These are 132 typhoon (or heavy rainfall) events from the past 31 years (1971-2001), published by the Water Resources Agency (WRA), Taiwan. A total of 8640 data sets are obtained. We believe these vast data sets are one of the most extensive and valuable event basis data sets for building an artificial neural network in the literature.

The 132 events data are divided into three independent subsets: the training, verification, and the testing subsets. The training subset includes 62 events $(4248$ data sets); the verification subset has 38 events (2064 data sets); while the testing subset has the remaining 32 events (2328 data sets) (Table 2). The ratio of training sets to verification sets to testing sets is approximately $2: 1: 1$. Obviously, training data set must cover all the characters of the problem, and it is desirable that training data includes all the maximal and minimal values. Moreover, in the process of segregation, the average and standard deviation of three independent subsets were computed to ensure the data sets were divided equally among three subsets.

First, the training subsets are repeatedly used to build networks and to adjust the connected weights of the constructed networks. Afterward, the verification subset is used to simulate the performance of the built models for checking its suitability of generalization, and the best network is selected for later use. The testing data set is then used for final evaluation of the selected network performance. It is worth mentioning that the testing sets must be unseen by model in training or verification phase.

\subsection{The forecasting models}

One of the most important tasks in developing a satisfactory forecasting model is the selection of the input variables, which determines the architecture of the model. In this study, our intent is to demonstrate that the neuro-fuzzy network has the ability to deal with human knowledge and enhance the model performance. We developed two ANFIS models for water level forecasting: one with a human decision (i.e. reservoir outflow) as input variable, another without. Because the flow of a river represents the total response (output) of a watershed to the hydrological input, for simplicity, we only use the water levels of the five upstream flow gauge stations as the rest of the inputs, the rainfall and other meteorological factors are not concerned as input.

Similar to most of the time-delay neural networks, which use time delays to learn the salient features of the input patterns and to perform temporal process, we also implemented three shifted inputs. Because the travel distances and times of flow from each station to the Shihmen reservoir are different, which are approximate from 1 to $4 \mathrm{~h}$, the travel times are taken into consideration for building the network and the shifted inputs of the five gauge stations are adopted. The model inputs and output can be represented as

Model (1) With upstream flow patterns and current outflow from reservoir as input variables

$$
\begin{aligned}
L_{\mathrm{m}}(t+i)= & f\left(L_{\mathrm{s}}(t-5), L_{\mathrm{s}}(t-4), L_{\mathrm{s}}(t-3), L_{\mathrm{y}}(t-4),\right. \\
& L_{\mathrm{y}}(t-3), L_{\mathrm{y}}(t-2), L_{\mathrm{l}}(t-3), L_{1}(t-2), \\
& L_{\mathrm{l}}(t-1), L_{\mathrm{g}}(t-2), L_{\mathrm{g}}(t-1), \\
& L_{\mathrm{g}}(t), L_{\mathrm{h}}(t-2), L_{\mathrm{h}}(t-1), L_{\mathrm{h}}(t), L_{\mathrm{m}}(t-1), \\
& \left.L_{\mathrm{m}}(t), O(t)\right)
\end{aligned}
$$

Model (2) With only upstream flow patterns (without reservoir outflow) as input variables

$$
\begin{aligned}
L_{\mathrm{m}}(t+i)= & f\left(L_{\mathrm{s}}(t-5), L_{\mathrm{s}}(t-4), L_{\mathrm{s}}(t-3), L_{\mathrm{y}}(t-4),\right. \\
& L_{\mathrm{y}}(t-3), L_{\mathrm{y}}(t-2), L_{\mathrm{l}}(t-3), L_{\mathrm{l}}(t-2), \\
& L_{\mathrm{l}}(t-1), L_{\mathrm{g}}(t-2), L_{\mathrm{g}}(t-1), \\
& L_{\mathrm{g}}(t), L_{\mathrm{h}}(t-2), L_{\mathrm{h}}(t-1), L_{\mathrm{h}}(t), L_{\mathrm{m}}(t-1), \\
& \left.L_{\mathrm{m}}(t)\right)
\end{aligned}
$$

where $L_{\mathrm{m}}(t+i)$ is the water level in station $\mathrm{m}$ at time $t+i$; the subscripts $\mathrm{s}, \mathrm{y}, 1, \mathrm{~g}, \mathrm{~h}$, and $\mathrm{m}$ represent gauge stations of the Shiouluan, Yufeng, Lingchiao, Gauyi, Shiayun, and Shihmen reservoir, respectively. Besides, $O(t)$ mean current outflow from reservoir. In all, there are 18 input variables in Model 1 (Fig. 3) and 17 input variables in Model 2. 


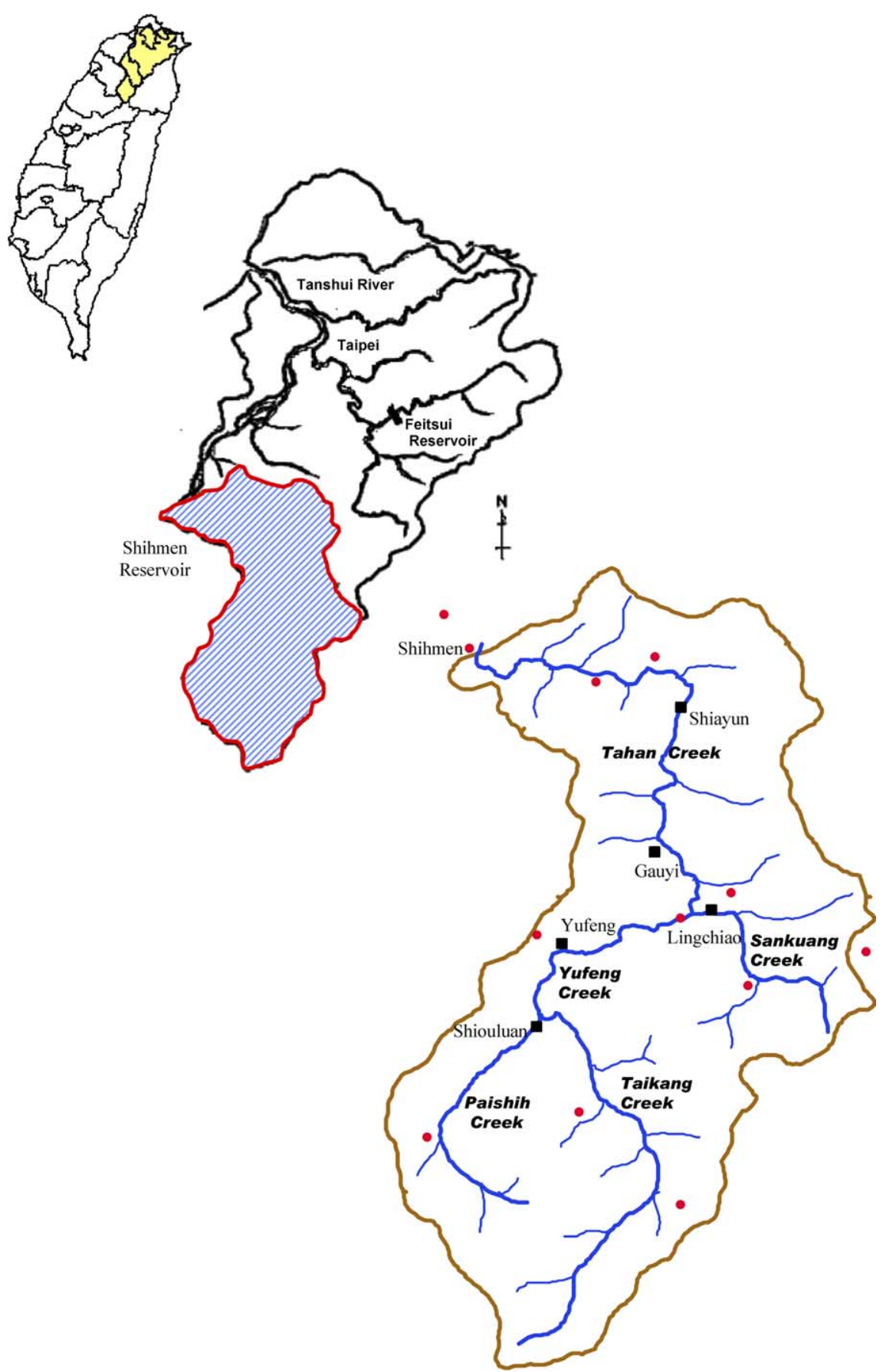

Fig. 2. Location of the Shihmen reservoir and gauge stations.

Table 1

Data of five gauge stations above Shihmen reservoir

\begin{tabular}{llll}
\hline Gauge station & Established year & Elevation $(\mathrm{m})$ & Basin area $\left(\mathrm{km}^{2}\right)$ \\
\hline Shiayun & 1957 & 246 & 623 \\
Gauyi & 1957 & 438 & 542 \\
Lingchiao & 1956 & 525 & 107 \\
Yufeng & 1956 & 684 & 335 \\
Shiouluan & 1956 & 827 & 115 \\
\hline
\end{tabular}

3.3. Establishment of fuzzy rule bases by the subtractive fuzzy clustering

The ANFIS models are built to create the fuzzy inference system and then to estimate the reservoir water level based on given input-output patterns. As mentioned above, there are 18 and 17 input variables for Models 1 and 2, respectively. In the ANFIS model, each input variable, which varies within a range, might be 
Table 2

The selection of three independent subsets

\begin{tabular}{llll}
\hline Set & Training & Verification & Testing \\
\hline Year & $1971,1976,1979,1982,1985,1986$, & $1972,1973,1978,1981,1984$, & $1975,1980,1983,1989$, \\
& $1988,1991,1995,1998,2001$ & $1987,1990,1994,19971999$ & $1992,1996,2000$ \\
Number of events & 62 & 38 & 32 \\
Data sets $_{\text {Mean }(m)^{\mathrm{a}}}$ & 4248 & 2064 & 2328 \\
${\text { Standard deviation }(\mathrm{m})^{\mathrm{a}}}^{\mathrm{a}}$ & 236.781 & 237.623 & 237.356 \\
\hline
\end{tabular}

${ }^{\text {a }}$ Water level in the reservoir.

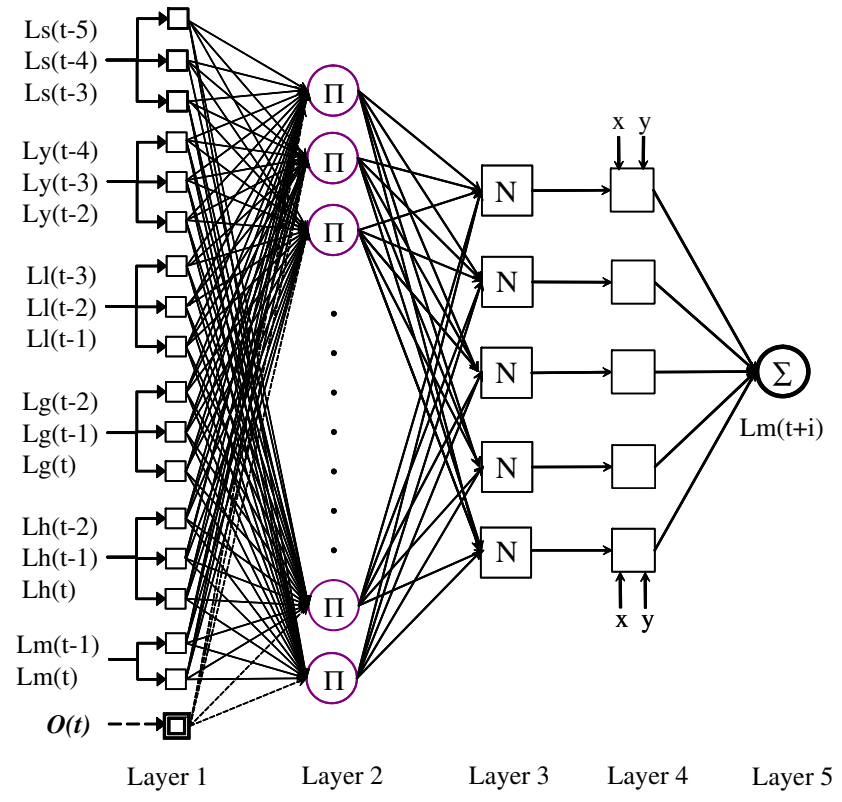

Fig. 3. The architecture of reservoir water level forecasting model.

clustered into several class values in layer 1 to build up fuzzy rules, and each fuzzy rule would be constructed through several parameters of membership function in layer 2. Accordingly, the number of parameters which need to be determined is enormous as the rule is increased. Since our input variables are quite large, i.e. 17 or 18, which can produce many possible combination conditions, the original ANFIS structure becomes discouraging. To solve this problem, we use subtractive fuzzy clustering to establish the rule base relationship between the input and output variables.
The subtractive fuzzy clustering can automatically determine the number of clusters. It assumes each data point is a potential cluster center and calculates a measure of the likelihood that each data point would define the cluster center, based on the density of surrounding data points.

First, each data point is considered as a potential cluster center instead of grid point. The density measure of each data point $x_{i}$ is defined as

$D_{i}=\sum_{j=1}^{n} \exp \left(-\frac{\left\|x_{i}-x_{j}\right\|^{2}}{\left(r_{a} / 2\right)^{2}}\right)$

where the positive constant $r_{a}$ is the radius defining a neighborhood of a cluster center. After the density measure of each data point is selected, the point with the highest density $D_{\mathrm{c} 1}$ as the first cluster center $x_{\mathrm{c} 1}$ is selected. In order to avoid neighborhood points of the first cluster center being selected as the second center, the density measure of each data point $x_{i}$ is revised as

$D_{i}=D_{i}-D_{\mathrm{cl}} \exp \left(-\frac{\left\|x_{i}-x_{\mathrm{cl}}\right\|^{2}}{\left(r_{b} / 2\right)^{2}}\right)$

A recommended value of setting $r_{b}$ is set equal to 1.5 $r_{a}$. The modification of the density measure in the remaining point set is applied in each step. The process of determining the cluster center and its corresponding density repeats until some stop conditions have been reached. The detailed algorithm and process of implementing the subtractive fuzzy clustering into ANFIS model can be found in Chiu [8] and our previous work [5]. After several numbers of clustering are evaluated, each forecasting model adopts appropriate number of rules (Table 3). It appears that the subtractive fuzzy

Table 3

The performance of fitted two ANFIS model at different phases

\begin{tabular}{|c|c|c|c|c|c|c|c|c|c|c|c|c|c|c|}
\hline & & \multirow{2}{*}{$\begin{array}{l}\text { Number } \\
\text { of rules }\end{array}$} & \multicolumn{4}{|c|}{ Training } & \multicolumn{4}{|c|}{ Verification } & \multicolumn{4}{|c|}{ Testing } \\
\hline & & & $G_{\text {bench }}$ & MAE & RMSE & $\mathrm{CC}$ & $G_{\text {bench }}$ & MAE & RMSE & $\mathrm{CC}$ & $G_{\text {bench }}$ & MAE & RMSE & $\mathrm{CC}$ \\
\hline \multirow[t]{3}{*}{ Model 1} & $t+1$ & 4 & 0.680 & 0.320 & 0.508 & 0.996 & 0.879 & 0.196 & 0.307 & 0.999 & 0.733 & 0.436 & 0.597 & 0.998 \\
\hline & $t+2$ & 9 & 0.696 & 0.418 & 0.726 & 0.993 & 0.532 & 0.525 & 0.875 & 0.993 & 0.625 & 0.741 & 1.007 & 0.994 \\
\hline & $t+3$ & 10 & 0.796 & 0.486 & 0.734 & 0.992 & 0.629 & 0.651 & 0.976 & 0.988 & 0.658 & 0.784 & 1.186 & 0.990 \\
\hline \multirow[t]{3}{*}{ Model 2} & $t+1$ & 7 & 0.530 & 0.393 & 0.616 & 0.995 & 0.544 & 0.369 & 0.597 & 0.997 & 0.611 & 0.484 & 0.720 & 0.996 \\
\hline & $t+2$ & 9 & 0.629 & 0.469 & 0.800 & 0.991 & 0.477 & 0.601 & 0.926 & 0.989 & 0.436 & 1.035 & 1.436 & 0.986 \\
\hline & $t+3$ & 10 & 0.693 & 0.574 & 0.900 & 0.989 & 0.549 & 0.720 & 1.075 & 0.985 & 0.486 & 1.148 & 1.652 & 0.976 \\
\hline
\end{tabular}


clustering does significantly reduce the number of rules, where all forecasting models only need a few number of rules. For example, the forecasting Model 1 for $t+1$ only has four rules and for $t+2$ only has nine rules. That means the vast and complex input-output patterns (18 input variables and one output) could be efficiently clustered into a few of rules, and those rules could make an accurate forecasting model.

\section{Results}

To assess the models' performances, several criteria are used and shown as below.

(1) Correlation coefficient $(C C)$. Indicates the strength of relationships between observed and estimated water level. The correlation coefficient is a number between 0 and 1 , and the higher the correlation coefficient the better

$$
\begin{aligned}
& \rho_{\hat{Q}, Q_{i}}=\frac{\operatorname{Cov}\left(\hat{Q}_{i}, Q_{i}\right)}{\rho_{\hat{Q}_{i}} \cdot \rho_{Q_{i}}} \text { where }-1 \leqslant \rho_{\hat{Q}_{i}, Q_{i}} \leqslant 1 \\
& \text { and } \operatorname{Cov}\left(\hat{Q}_{i}, Q_{i}\right)=\frac{1}{n} \sum_{i=1}^{n}\left(\hat{Q}_{i}-\mu_{\hat{Q}_{i}}\right)\left(Q_{i}-\mu_{Q_{i}}\right)
\end{aligned}
$$

The variables $\mu, \rho, n$ means the average of the variable, the standard deviation of the variable and the number of data point, respectively.

(2) Root mean square error (RMSE). Evaluates the residual between observed and forecasted water level. This index assumes that larger forecast errors are of greater importance than smaller ones; hence they are given a more than proportionate penalty

$$
\mathrm{RMSE}=\left[\sum_{i=1}^{n} \frac{\left(\hat{Q}_{i}-Q_{i}\right)^{2}}{n}\right]^{0.5}
$$

Note that RMSE equal to zero represents perfect fit.

(3) Mean absolute error ( $M A E$ ). A weighted average of the absolute errors and the lower the MAE the better

$$
\mathrm{MAE}=\sum_{i=1}^{n} \frac{\left|\hat{Q}_{i}-Q_{i}\right|}{n}
$$

(4) $G_{\text {bench. }}$. Compute the goodness-of-fit measures [20]

$G_{\text {bench }}=1-\frac{\sum_{i=1}^{n}\left(\hat{Q}_{i}-Q_{i}\right)^{2}}{\sum_{i=1}^{n}\left(\hat{Q}_{i}-Q_{i, \text { bench }}\right)^{2}}$

Here, $\hat{Q}_{i}$ and $Q_{i}$ represent the forecasted and observed water level, respectively. $Q_{i \text {,bench }}$ is the pre- vious observed value, ex. for $n$ step ahead prediction $Q_{i \text {,bench }}=Q_{i-n}$.

If $G_{\text {bench }}$ is negative, the forecasting performance is poorer than the benchmark.

If $G_{\text {bench }}$ is equal to zero, the performance is as good as the benchmark.

If $G_{\text {bench }}$ is equal to one, it means perfect fit.

\subsection{The simulation results of two ANFIS models}

The ANFIS models are compared based on their performance in (1) training sets, (2) verification sets, and (3) testing sets. The results are summarized in Table 3. It appears that the ANFIS models are accurate and consistent in different subsets, where all the values of RMSE and MAE are smaller, and all correlation coefficients are also very close to unity. It also shows that the forecasting Model 1 results in a much lower value of the MAE and RMSE and higher value of the $G_{\text {bench }}$ and $\mathrm{CC}$ than Model 2. Model 1, which adds the outflow from reservoir as input variable, has consistently superior performance with regard to all indexes over Model 2. We believe this is mainly because the reservoir is a control system which can be operated by human decisions through their knowledge and/or experience, and the water level of the reservoir can be significantly changed within a short period (a few hours in our cases). Therefore, the forecasting model which includes this important factor would be more accurate. These results might also suggest that the ANFIS has a great ability to learn from input-output patterns, which represent the watershed's physiographic and hydrometeorologic lumped effects and human knowledge.

Overall, the performance of the two ANFIS models is very good. The results demonstrate that the ANFIS can be successfully applied to establish the forecasting models that could provide accurate and reliable 1-3 hours-ahead water level prediction.

Fig. 4 shows the observed and forecasted reservoir water level of one, two and three step ahead, respectively, by the ANFIS Models 1 and 2 in test phases. The figures nicely demonstrate that (1) the models' performances are, in general, accurate, where all data points roughly fall onto the line of agreement; (2) Model 1 is consistently superior to Model 2 in test phases; and (3) the predicting accuracy will be decreased as predicting time-step increase.

To get a brief picture of the general performance of the constructed model, we also provide the hydrographs of observed water level and one-step ahead, two-step ahead and three-step ahead water level predictions of Shihmen reservoir for one of the test phase events (Bilisz typhoon in 2000) in Fig. 5. Again, it indicates that the model can nicely forecast the 1-3 hours-ahead water levels during a typhoon event. 
MODEL 1
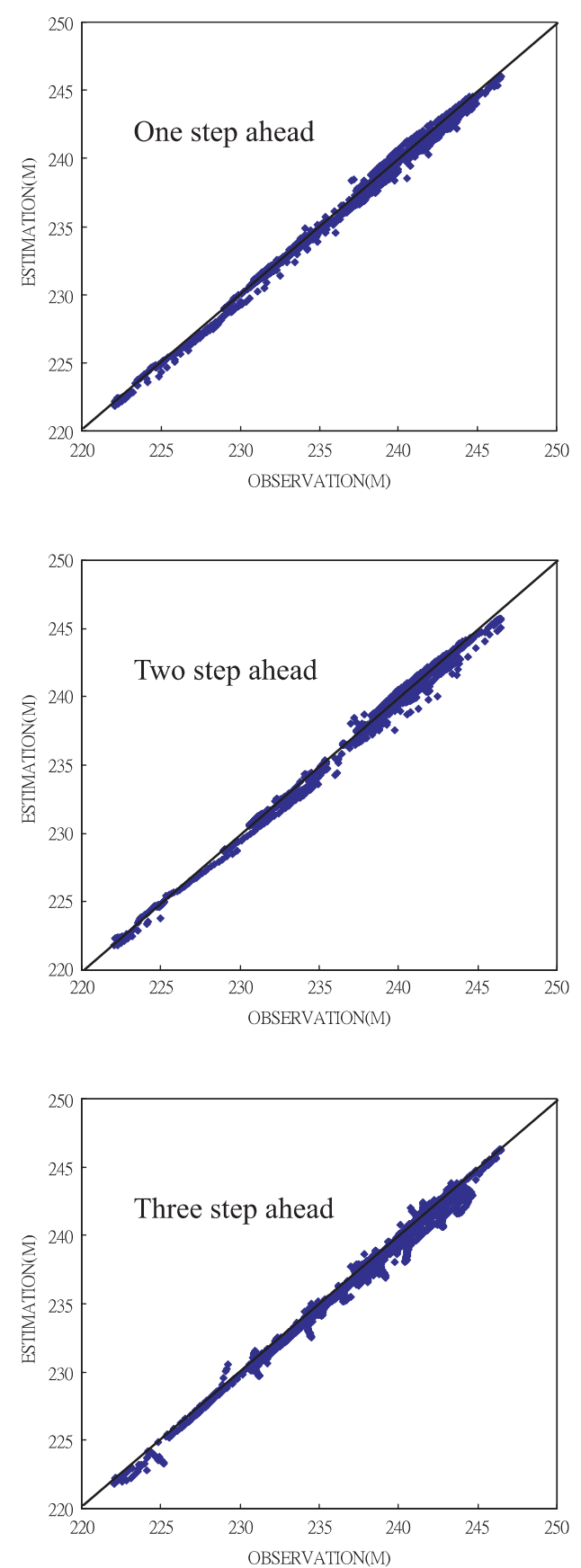

MODEL 2
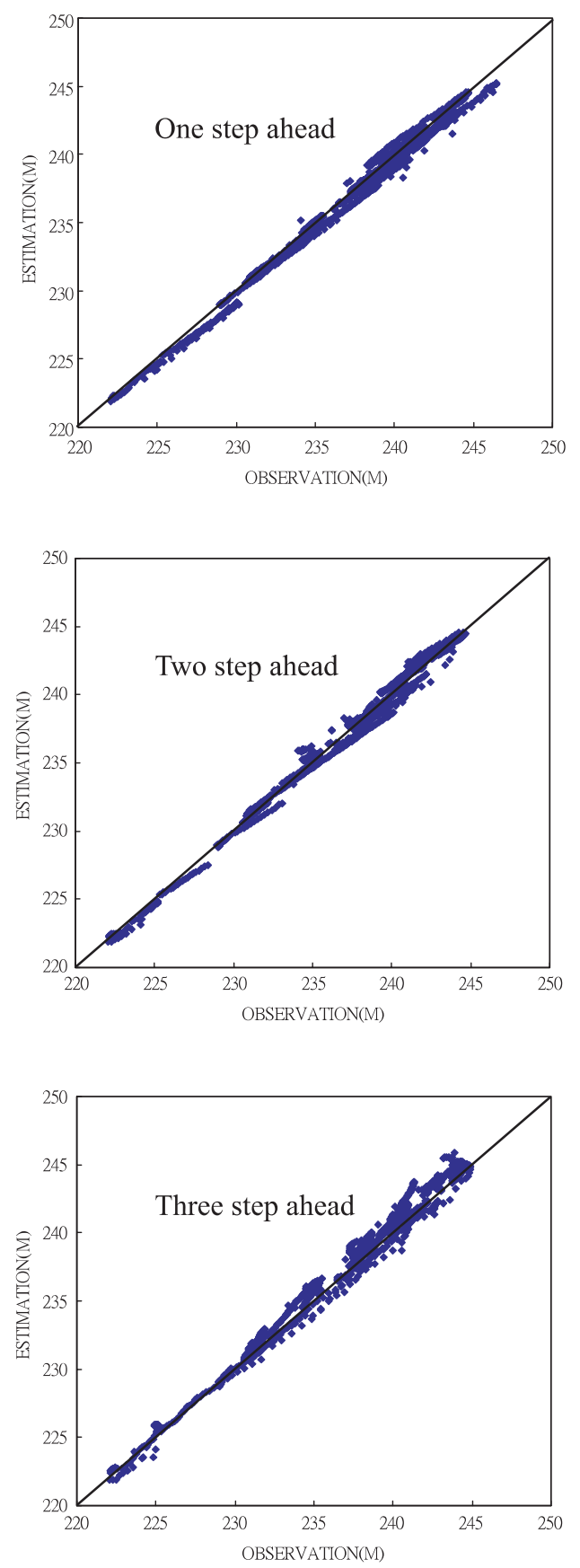

Fig. 4. Comparison of observed and forecasted values of Models 1 and 2 for one-three steps ahead prediction in test phase.

\section{Conclusions}

In this study, we propose the use of a novel neurofuzzy model, the adaptive network-based fuzzy inference system (ANFIS), to construct 1-3 hours-ahead water level forecasting system to insure reservoir safety, minimizing the damage resulting from a natural disaster, and sufficiently make use of available water resources, ANFIS is a powerful fuzzy logic neural network, which provides a method for fuzzy modeling to learn information about the data set that best allow the associated fuzzy inference system to trace the given input/output data.

The applicability and capability of the ANFIS model are investigated through the use of great number data sets in the Shihmen reservoir, Taiwan. The total number of typhoon or heavy rainfall events is 132 , which include 8640 hourly data sets. The historical data sets are di- 

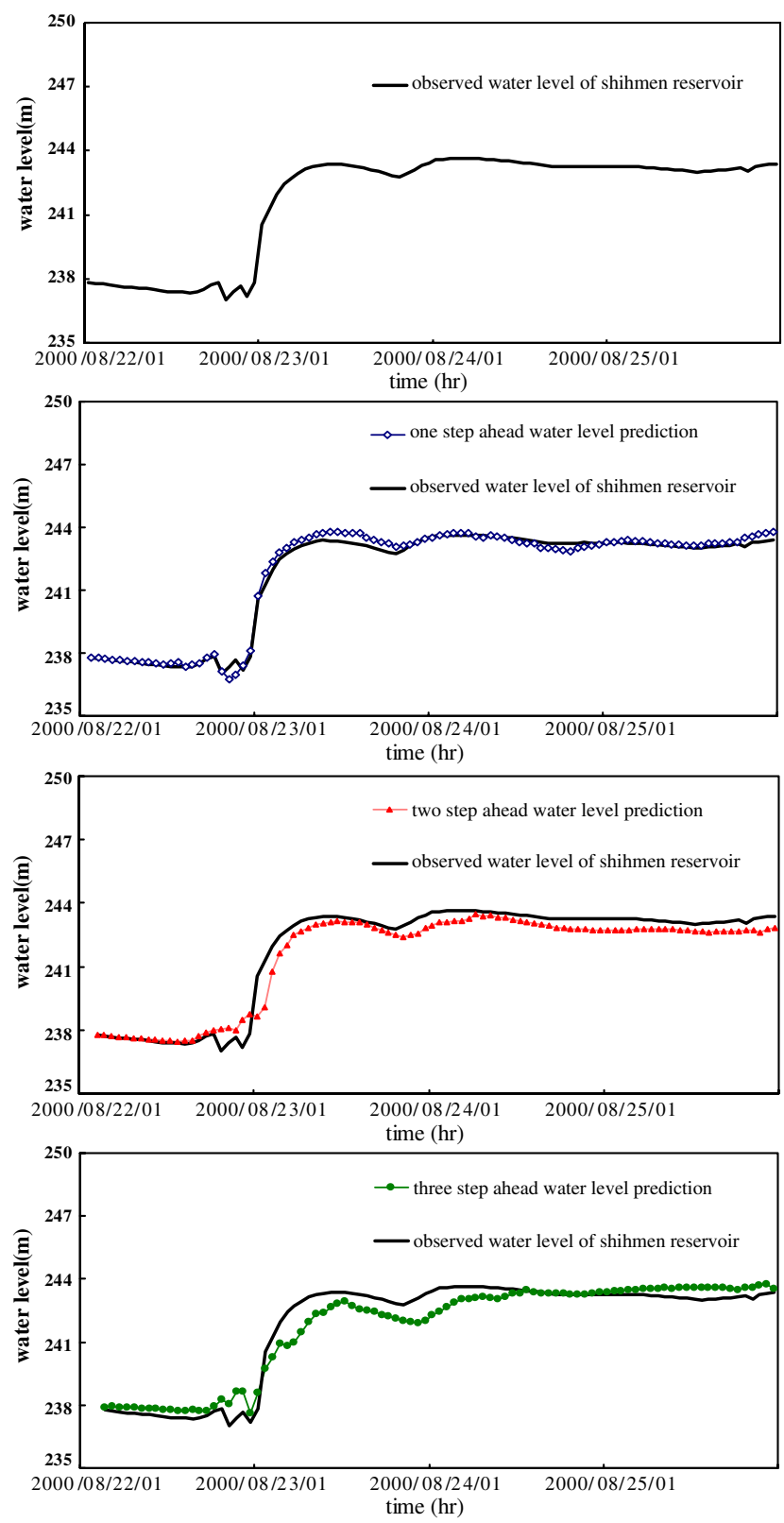

Fig. 5. Observed water level and one to three steps ahead water level predictions of Shihmen reservoir in testing phase in 2000 (Bilisz typhoon 2000/08/22-08/25).

vided into three independent sets to train, to verify and to test the constructed models. Prediction of reservoir water level might be significantly different from prediction of a streamflow, because the variability of a reservoir cannot be solely determined by hydrological effects. The human operating decision could significantly change the status of water level within a short period; consequently, a suitable prediction model should include human decisions. In order to demonstrate that the neuro-fuzzy network has the ability to deal with human knowledge and enhance the model performance, two ANFIS models were developed and investigated: one with a human decision, reservoir outflow, as input variable, another without. It appears that the model with human decision as input variable has consistently superior performance with regard to all used indexes than the model without this important input.

In general, the ANFIS models provide accurate and reliable water level prediction for next three time steps, where the correlation coefficients (CC) are very close to unity (larger than 0.99) in most of cases. We conclude that the constructed ANFIS models, through the subtractive fuzzy clustering, can efficiently deal with vast and complex input-output patterns, and has a great ability to learn and build up a neuro-fuzzy inference system for prediction, and the forecasting results provide a useful guidance or reference for flood control operations.

\section{Acknowledgements}

This paper is based on partial work supported by National Science Council, R.O.C. (Grant No. NSC912313-B-002-315). We would like to thank Assistant Professor Li-Chiu Chang (Tamkang University) for her valuable suggestion and assistance in this study. The data provided by the Water Resources Agency, Taiwan, are also great appreciated. In addition, the authors are indebted to the reviewers for their valuable comments and suggestions.

\section{References}

[1] Altug S, Chow MY, Trussell HJ. Fuzzy inference systems implemented on neural architectures for motor fault detection and diagnosis. IEEE Trans Ind Electron 1999;46(6):1069-79.

[2] Baratti R, Cannas B, Fanni A, Pintus M, Sechi GM, Toreno N. River flow forecast for reservoir management through neural networks. Neurocomputing 2003;55(3-4):421-37.

[3] Chang FJ, Chang LC, Huang HL. Real time recurrent neural network for streamflow forecasting. Hydrol Process 2002;16: 2577-88.

[4] Chang FJ, Chen YC. A counterpropagation fuzzy-neural network modeling approach to real time stream flow prediction. J Hydrol 2001;245:153-64.

[5] Chang LC, Chang FJ. Intelligent control of modeling of real time reservoir operation. Hydrol Process 2001;15(9):1621-34.

[6] Chang LC, Chang FJ, Chiang YM. A two-step-ahead recurrent neural network for stream-flow forecasting. Hydrol Process 2004; 18(1):81-92.

[7] Chang YT, Chang LC, Chang FJ. Intelligent control for modeling of real-time reservoir operation-part II: ANN with operating rule curves. Hydrol Process 2005;19:1431-44.

[8] Chiu SL. Fuzzy model identification based on cluster estimation. J Intell Fuzzy Syst 1994;2(3):267-78.

[9] Croley II TE. Using meteorology probability forecasts in operational hydrology. Reston: ASCE Press; 2000.

[10] Dawson CW, Wilby RL. An artificial neural network approach to rainfall-runoff modeling. Hydrol Sci 1998;43(1):47-67.

[11] Day GN. Extended streamflow forecasting using the NWSRFS. J Water Resour Plan Manage 1985;111(2):157-70. 
[12] Djukanovic MB, Calovic MS, Vesovic BV, Sobajic DJ. Neurofuzzy controller of low head hydropower plants using adaptivenetwork based fuzzy inference. IEEE Trans Energy Conver 1997;12(4):375-81.

[13] French MN, Krajewski WF, Cuykendall RR. Rainfall forecasting in space and time using a neural network. J Hydrol 1992;137:1-31.

[14] Govindaraju RS, Rao AR. Artificial neural networks in hydrology. Dordrecht: Kluwer Academic Publishers; 2000.

[15] Grimes DIF, Coppola E, Verdecchia M, Visconti G. A neural network approach to real-time rainfall estimation for Africa using satellite data. J Hydrometeorol 2003;4:1119-33.

[16] Ham FM, Kostanic I. Principles of neurocomputing for science and engineering. New York: McGraw Hill; 2001.

[17] Hasebe M, Nagayama Y. Reservoir operation using the neural network and fuzzy systems for dam control and operation support. Adv Eng Software 2002;33(5):245-60.

[18] Hsu KL, Gupta HV, Sorooshian S. Artificial neural network modeling of the rainfall-runoff process. Water Resour Res 1995; 31(10):2517-30.

[19] Jain SK, Das A, Srivastava DK. Application of ANN for reservoir inflow prediction and operation. J Water Resour Plan Manage 1999;125(5):263-71.

[20] Jan S. On the need for benchmarks in hydrological modeling. Hydrol Proces 2001;15:1063-4.

[21] Jang JSR. ANFIS: adaptive-network-based fuzzy inference system. IEEE Trans Syst, Man, Cybernet 1993;23(3):665-85.

[22] Karunanithi N, Grenney WJ, Whitley D. Neural network for river flow prediction. J Comput Civil Eng 1994;8:201-20.

[23] Labadie JW. Optimal operation of multireservoir systems: stateof-the-art review. J Water Resour Plan Manage 2004;130(2): 93-111.

[24] Lallahem S, Mania J. Evaluation and forecasting of daily groundwater outflow in a small chalky watershed. Hydrol Process 2003;17(8):1561-77.

[25] Liao HP, Su JP, Wu HM. An application of ANFIS to modeling of a forecasting system for the demand of teacher human resources. J Educat Psychol 2001;24(1):1-17.

[26] Luk KC, Ball JE, Sharma A. A study of optimal model lag and spatial inputs to artificial neural network for rainfall forecasting. $J$ Hydrol 2000;227:56-65.

[27] Maier H, Dandy G. Neural networks for the prediction and forecasting of water resources variables: a review of modeling issues and applications. Environ Model Software 2000;5(1): 101-24.

[28] Oonsivilai A, El-Hawary ME. Power system dynamic load modeling using adaptive-network-based fuzzy inference system. In Proceedings of the 1999 IEEE Canadian Conference on Electrical and Computer Engineering, Shaw Conference Center, Edmonton, Alberta, Canada, 1999.

[29] Ponnambalam K, Karray F, Mousavi SJ. Minimizing variance of reservoir systems operations benefits using soft computing tools. Fuzzy Sets Syst 2003;139:451-61.

[30] Raman H, Chandramouli V. Deriving a general operating policy for reservoirs using neural network. J Water Resour Plan Manage 1996;122(5):342-7.

[31] Russell SO, Campbell PF. Reservoir operating rules with fuzzy programming. J Water Resour Plan Manage 1996;122(3):165-70.

[32] Salas JD et al. Applied modeling of hydrologic time series. Water Resources Publications; 1985.

[33] Sfetsos A. A comparison of various forecasting techniques applied to mean hourly wind speed time series. Renewable Energy 2000;21:23-35.

[34] Shamseldin AY. Application of a neural network technique to rainfall-runoff modeling. J Hydrol 1997;199:272-94.

[35] Takagi T, Sugeno M. Fuzzy identification of systems and its applications to modeling and control. IEEE Trans Syst, Man, Cybernet 1985;15:116-32.

[36] Xu ZX, Li JY. Short-term inflow forecasting using an artificial neural network model. Hydrol Process 2002;16:2433-9.

[37] Yang CC, Prasher SO, Lacroix R, Sreekanth S, Patni NK, Masse L. Artificial neural network model for subsurfacedrained farmland. J Irrigat Drainage Eng 1997;123:285-92.

[38] Zadeh LA. Fuzzy Sets. Inform Contr 1965;8:338-53.

[39] Zhu ML, Fujita M. Comparisons between fuzzy reasoning and neural network methods to forecast runoff discharge. J Hydrosci Hydraul Eng 1994;12(2):131-41.

[40] ASCE Task Commitee on Application of Artificial Neural Networks in Hydrology. Artificial neural networks in hydrology I: Preliminary concepts. Journal of Hydrologic Engineering 2000;5(2):115-23.

[41] ASCE Task Commitee on Application of Artificial Neural Networks in Hydrology. Artificial neural networks in hydrology II: Hydrologic applications. Journal of Hydrologic Engineering 2000;5(2):124-37. 